\title{
RADIAL SOLUTIONS FOR A NONLOCAL BOUNDARY VALUE PROBLEM
}

\author{
RICARDO ENGUIÇA AND LUÍS SANCHEZ
}

Received 23 August 2005; Revised 20 December 2005; Accepted 22 December 2005

We consider the boundary value problem for the nonlinear Poisson equation with a nonlocal term $-\Delta u=f\left(u, \int_{U} g(u)\right),\left.u\right|_{\partial U}=0$. We prove the existence of a positive radial solution when $f$ grows linearly in $u$, using Krasnoselskii's fixed point theorem together with eigenvalue theory. In presence of upper and lower solutions, we consider monotone approximation to solutions.

Copyright (c) 2006 R. Enguiça and L. Sanchez. This is an open access article distributed under the Creative Commons Attribution License, which permits unrestricted use, distribution, and reproduction in any medium, provided the original work is properly cited.

\section{Introduction}

Let us consider the following nonlocal BVP in a ball $U=B(0, R)$ of $\mathbb{R}^{n}$ :

$$
\begin{gathered}
-\Delta u=f\left(u, \int_{U} g(u)\right), \\
\left.u\right|_{\partial U}=0
\end{gathered}
$$

where $f$ and $g$ are continuous functions. For simplicity we shall take $R=1$. We want to study the existence of positive radial solutions

$$
u(x)=v(\|x\|)
$$

of (1.1). This may be seen as the stationary problem corresponding to a class of nonlocal evolution (parabolic) boundary value problems related to relevant phenomena in engineering and physics. The literature dealing with such problems has been growing in the last decade. The reader may find some hints on the motivation for the study of this mathematical model, for example, in the paper by Bebernes and Lacey [1]. For more recent developments, see [2] and the references therein. 
Here we are considering a nonlocal term inserted in the right-hand side of the equation. Note, however, that it is also of interest to study boundary value problems where the nonlocal expression appears in a boundary condition. We refer the reader to the recent paper by Yang [13] and its references.

When dealing with a nonlinear term with rather general dependence on the nonlocal functional as in (1.1) new difficulties arise with respect to the treatment of standard boundary value problems. Differences of behaviour which are met in general elliptic and parabolic problems are already present in simple models as those we shall analyse in this paper. For instance, the use of the powerful lower and upper solution method (good accounts of which can be consulted in the monographs of Pao [10] and De Coster and Habets [3]) is limited by the absence of general maximum principles. Even for linear problems with nonlocal terms the issue of positivity is far from trivial and may require a detailed study via the analysis of the Green's operator, as in Freitas and Sweers [6].

The purpose of this paper is twofold. First, we want to improve a quite recent result of Fijałkowski and Przeradzki [5]: these authors have obtained existence of positive radial solutions of (1.1) by using Krasnoselskii's fixed point theorem in cones; the main assumption is that $f$ may grow at most like $A u+B$, the bound on $A$ being computed by means of a Green's function. By using a similar theoretical background, together with the consideration of the eigenvalues of the underlying linear problem, we show that an improvement of that bound is possible. This is done in Theorem 3.2. Second, while remaining in the same simple general setting, we will handle (1.1) from the point of view of the upper and lower solution method. We establish a nonlocal maximum principle (Lemma 4.6) and we use it as a device to obtain a monotone approximation scheme for the radial solutions of (1.1) in presence of lower and upper solutions (Theorem 4.10). We follow an idea used by Jiang et al. [9] in studying a fourth-order periodic problem.

Note that we could use similar methods to consider the case where $U=B(0,1) \backslash \bar{B}(0, \rho)$, with $0<\rho<1$. Similar results could then be reached. We remark also that for special classes of functions $f$ and $g$ different approaches are needed. For instance, in [8] variational methods have been used to study existence and multiplicity when $f(u, v)=g(u)$ / $v^{P}(p>0)$ and $g$ behaves as an exponencial function.

The authors wish to thank the referee for carefully reading the manuscript and hints to improve its final form.

\section{Some auxiliary results}

It is well known that the existence of a solution for some boundary value problems is equivalent to the existence of a fixed point of a certain operator. For our purpose we need to consider a second-order ordinary differential equation of the form

$$
-\left(p(t) u^{\prime}(t)\right)^{\prime}=p(t) f(t, u(t))
$$

with boundary conditions

$$
u^{\prime}(0)=u(1)=0
$$


where $f$ is a continuous function in $[0,1] \times \mathbb{R}$ and $p \in C[0,1]$ is positive and increasing in ] $0,1]$. If $p>0$ in $[0,1]$, it is well known that the problem is fully regular, having a standard reduction to a fixed point problem:

$$
u=T f(\cdot, u(\cdot)) \quad \text { in } C[0,1]
$$

where $T$ is the linear operator that takes $v \in C[0,1]$ into the unique solution $u$ of

$$
-\left(p(t) u^{\prime}(t)\right)^{\prime}=p(t) v(t), \quad u^{\prime}(0)=u(1)=0 .
$$

In addition we can write explicitly

$$
T v(t)=\int_{0}^{1} G(t, s) v(s) d s,
$$

where $G(t, s)$ is the Green's function associated to the problem. The Green's function is continuous in $[0,1] \times[0,1]$, so $T$ is a completely continuous linear operator in $C[0,1]$.

We are interested in the case where $p(t)>0$ in $] 0,1]$ only. Under certain assumptions we still have a continuous Green's function for the linear problem (2.4). The reader can find a more general approach in [7], but for completeness we include here a simple version which is sufficient for our purpose.

Lemma 2.1. Let $p$ be continuous, increasing in [0,1], $p(0)=0$ and $p>0$ in $] 0,1]$. If the function

$$
p(s) \int_{s}^{1} \frac{1}{p(\tau)} d \tau
$$

is continuously extendible to $[0,1]$, then the operator $T: C[0,1] \rightarrow C[0,1]$ previously considered is well defined, linear, and completely continuous.

Proof. Consider the equation

$$
-\left(p(t) u^{\prime}(t)\right)^{\prime}=p(t) v(t)
$$

with boundary conditions (2.2). Integrating both sides we get

$$
-p(t) u^{\prime}(t)=\int_{0}^{t} p(s) v(s) d s .
$$

Integrating again, we obtain

$$
\begin{aligned}
u(t) & =\int_{t}^{1} \frac{d \tau}{p(\tau)} \int_{0}^{\tau} p(s) v(s) d s \\
& =\int_{0}^{t} p(s) v(s) d s \int_{t}^{1} \frac{1}{p(\tau)} d \tau+\int_{t}^{1} p(s) v(s) d s \int_{s}^{1} \frac{1}{p(\tau)} d \tau \\
& =\int_{0}^{1} G(t, s) v(s) d s
\end{aligned}
$$


4 Radial solutions for a nonlocal boundary value problem

where

$$
G(t, s)= \begin{cases}p(s) \int_{t}^{1} \frac{1}{p(\tau)} d \tau, & t \geq s \\ p(s) \int_{s}^{1} \frac{1}{p(\tau)} d \tau, & t \leq s\end{cases}
$$

is clearly continuous in $[0,1] \times[0,1]$, so that the operator

$$
T v(t)=\int_{0}^{1} G(t, s) v(s) d s=\int_{0}^{t} p(s) \int_{t}^{1} \frac{1}{p(\tau)} d \tau v(s) d s+\int_{t}^{1} p(s) \int_{s}^{1} \frac{1}{p(\tau)} d \tau v(s) d s
$$

is completely continuous in $C[0,1]$.

It is trivial to see that $T v(1)=0$ and if we differentiate the expression for $T v(t)$ we obtain

$$
\begin{aligned}
(T v)^{\prime}(t) & =p(t) \int_{t}^{1} \frac{1}{p(\tau)} d \tau v(t)+\int_{0}^{t}-\frac{p(s) v(s)}{p(t)} d s-p(t) \int_{t}^{1} \frac{1}{p(\tau)} d \tau v(t) \\
& =-\int_{0}^{t} \frac{p(s) v(s)}{p(t)} d s
\end{aligned}
$$

and thus

$$
(T v)^{\prime}(0)=\lim _{t \rightarrow 0}-\int_{0}^{t} \frac{p(s) v(s)}{p(t)} d s=-\lim _{t \rightarrow 0} v(0) \frac{\int_{0}^{t} p(s)}{p(t)}=0
$$

Remark 2.2. The continuous functions $p(t)=t^{n}$, with $n>0$, satisfy the assumptions of the lemma. [4]).

The following fixed point theorem of Krasnoselskii will be used in the next section (see

Theorem 2.3. Let $P$ be a cone in a Banach space and $S: P \rightarrow P$ a completely continuous operator. If there exist positive constants $r<R$ such that (compression case)

$\|S x\| \geq\|x\|, \quad \forall x \in P$ such that $\|x\|=r, \quad\|S x\| \leq\|x\|, \quad \forall x \in P$ such that $\|x\|=R$,

then S has a fixed point $x$ in $P$ such that $r<\|x\|<R$.

\section{Nonlinearities with linear growth in $u$ : a positive solution}

Let $f: \mathbb{R}^{+} \times \mathbb{R} \rightarrow \mathbb{R}^{+}$and $g: \mathbb{R}^{+} \rightarrow \mathbb{R}$ be continuous functions. The radial solutions $v$ of the problem (1.1) solve the ordinary differential equation

$$
-v^{\prime \prime}(r)-\frac{n-1}{r} v^{\prime}(r)=f\left(v(r), \omega_{n} \int_{0}^{1} s^{n-1} g(v(s)) d s\right)
$$


which is equivalent to

$$
-\left(r^{n-1} v^{\prime}(r)\right)^{\prime}=r^{n-1} f\left(v(r), \omega_{n} \int_{0}^{1} s^{n-1} g(v(s)) d s\right),
$$

with boundary conditions

$$
\lim _{r \rightarrow 0^{+}} v^{\prime}(r)=v(1)=0,
$$

where $\omega_{n}$ is the measure of the unit sphere in $\mathbb{R}^{n}$.

The homogeneous equation $-v^{\prime \prime}-(n-1) v^{\prime} / r=0$, with the boundary conditions (3.3), has only the trivial solution, and therefore there exists a Green's function associated to the linear problem. In fact, the Green's function may be written according to Lemma 2.1 (see also [5]):

(i) for $n>2$,

$$
G(r, t)=\frac{t^{n-1}}{n-2}\left(\frac{1}{\max (r, t)^{n-2}}-1\right)
$$

(ii) and for $n=2$,

$$
G(r, t)=-t \ln (\max (r, t)) .
$$

Hence the boundary value problem (3.1)-(3.3) is equivalent to the integral equation

$$
v(r)=\int_{0}^{1} G(r, t) f\left(v(t), \omega_{n} \int_{0}^{1} s^{n-1} g(v(s)) d s\right) d t .
$$

In $C[0,1]$, the Banach space of continuous functions in $[0,1]$ with the usual norm, let $P$ be the cone of the nonnegative functions. The radial solutions of (1.1) are exactly the fixed points of the completely continuous operator $S: P \rightarrow P$, defined by

$$
S(v)(r)=\int_{0}^{1} G(r, t) f\left(v(t), \omega_{n} \int_{0}^{1} s^{n-1} g(v(s)) d s\right) d t .
$$

In [5], the following theorem is proved.

Theorem 3.1. Let $f: \mathbb{R}^{+} \times \mathbb{R} \rightarrow \mathbb{R}^{+}$and $g: \mathbb{R}^{+} \rightarrow \mathbb{R}$ be continuous functions, and

$$
\gamma=\sup _{r \in[0,1]} \int_{0}^{1} G(r, s) d s
$$

Suppose there exist constants $A, B \in \mathbb{R}$ such that $0 \leq A<\gamma^{-1}$ and

$$
f(v, y) \leq A v+B
$$

for all $v \geq 0$ and $y \in \mathbb{R}$.

Then the problem (1.1) has a positive radial solution. 
6 Radial solutions for a nonlocal boundary value problem

We will show that the estimate on the constant $A$ in the previous result can be improved.

Consider the problem (3.1)-(3.3) and the associated eigenvalue problem:

$$
-v^{\prime \prime}(r)-\frac{n-1}{r} v^{\prime}(r)=\lambda v(r), \quad \text { with } \lim _{r \rightarrow 0^{+}} v^{\prime}(r)=0, \quad v(1)=0 .
$$

We have

$$
-v^{\prime \prime}(r)-\frac{n-1}{r} v^{\prime}(r)=\lambda v(r) \Longleftrightarrow\left(r^{n-1} v^{\prime}(r)\right)^{\prime}+\lambda r^{n-1} v(r)=0 .
$$

To find the eigenvalues, it is useful to consider the auxiliar initial value problem:

$$
\left(r^{n-1} v^{\prime}(r)\right)^{\prime}+r^{n-1} v(r)=0, \quad v(0)=1, \quad v^{\prime}(0)=0
$$

The solution $v$ to this problem is well defined in $\left[0,+\infty\left[\right.\right.$, oscillates, and has zeros $\left\{\xi_{n} \mid\right.$ $n \in \mathbb{N}\}$ such that $0<\xi_{1}<\xi_{2}<\cdots \rightarrow+\infty$, with $\xi_{n+1}-\xi_{n} \rightarrow \pi$ (see [12]).

Define $u(r)=v(\beta r)$. Then

$$
u^{\prime}(r)=\beta v^{\prime}(\beta r), \quad u^{\prime \prime}(r)=\beta^{2} v^{\prime \prime}(\beta r) .
$$

Using (3.12) we have

$$
(n-1)(\beta r)^{n-2} v^{\prime}(\beta r)+(\beta r)^{n-1} v^{\prime \prime}(\beta r)+(\beta r)^{n-1} v(\beta r)=0 \Longleftrightarrow\left(r^{n-1} u^{\prime}(r)\right)^{\prime}+\beta^{2} r^{n-1} u(r)=0 .
$$

It is obvious that $u^{\prime}(0)=0$, so it remains to find $\beta$ such that $u(1)=0$. As $u(1)=v(\beta)$, we get $\beta=\xi_{n}$ for some $n \in \mathbb{N}$, hence $\beta=\xi_{n}$ and, therefore, the eigenvalues of (3.10) are

$$
\lambda_{n}=\beta^{2}=\xi_{n}^{2} .
$$

Let us identify the zeros of the unique solution of (3.12). We have

$$
\left(r^{n-1} v^{\prime}(r)\right)^{\prime}+r^{n-1} v(r)=0 \Longleftrightarrow r^{n-3}\left(r^{2} v^{\prime \prime}+(n-1) r v^{\prime}+r^{2} v\right)=0,
$$

and the last equation has the form

$$
t^{2} u^{\prime \prime}+a t u^{\prime}+\left(b+c t^{m}\right) u=0,
$$

which is easily reduced to a Bessel equation (cf. [11]). Using the new independent variable

$$
y=r^{(n-2) / 2} v
$$

we obtain the transformed equation

$$
r^{2} y^{\prime \prime}+r y^{\prime}+\left(r^{2}-\left(\frac{n-2}{2}\right)^{2}\right) y=0
$$


whose solutions are well known, and thus we get

(i) $v(r)=c_{1} r^{(-n-2) / 2} J_{(n-2) / 2}(r)+c_{2} r^{(-n-2) / 2} K_{(n-2) / 2}(r)$ if $n$ is even, or

(ii) $v(r)=c_{1} r^{(-n-2) / 2} J_{(n-2) / 2}(r)+c_{2} r^{(-n-2) / 2} J_{(2-n) / 2}(r)$ if $n$ is odd,

where $c_{1}, c_{2}$ are constants and $J_{i}, K_{i}$ are Bessel functions of order $i$, of the first and second kind, respectively.

Taking into consideration the boundary conditions, the constant $c_{2}$ must be zero in both cases (otherwise we would have $\lim _{r \rightarrow 0^{+}} v(r)=\infty$ ), so that

$$
v(r)=c_{1} r^{(-n-2) / 2} J_{(n-2) / 2}(r)
$$

For our boundary value problem we know that $\gamma^{-1}=2 n$ (see [5]). If we compare $\sqrt{2 n}$ with $\xi_{1}$ - the zeros of these Bessel functions are well known-we can see that

$$
\sqrt{2 n}<\xi_{1}
$$

and hence,

$$
\gamma^{-1}<\lambda_{1} \quad \text { (first eigenvalue of (3.10)) }
$$

For instance, for $n=2$ or $n=4$ we have

$$
\begin{aligned}
& \sqrt{4}=2,000<\xi_{1}\left(J_{0}\right) \approx 2,404, \\
& \sqrt{8} \approx 2,828<\xi_{1}\left(J_{1}\right) \approx 3,832 .
\end{aligned}
$$

By adapting the approach of [5] we will prove the following improved version of Theorem 3.1 .

Theorem 3.2. Let $f: \mathbb{R}^{+} \times \mathbb{R} \rightarrow \mathbb{R}^{+}$and $g: \mathbb{R}^{+} \rightarrow \mathbb{R}$ be continuous functions, and $\lambda_{1}$ defined as above.

Suppose there exist constants $A, B \in \mathbb{R}$ such that $0 \leq A<\lambda_{1}$, and

$$
f(v, y) \leq A v+B, \quad \forall v \geq 0, y \in \mathbb{R} .
$$

Then the problem (1.1) has a positive radial solution.

Let $\phi$ be an eigenfunction associated with the first eigenvalue $\lambda_{1}$. We have

$$
-\phi^{\prime \prime}-\frac{n-1}{r} \phi^{\prime}=\lambda_{1} \phi, \quad \phi^{\prime}(0)=0=\phi(1) .
$$

Since our computation above shows that we may assume that $\phi(t)=v\left(\xi_{1} r\right)$ where $v(r)=r^{-n-2 / 2} J_{n-2 / 2}(r)$, it is clear that $\phi>0$ in $\left[0,1\left[\right.\right.$, (and, by the way, $\left.\phi^{\prime}(1)<0\right)$. We may therefore consider the norm

$$
\|v(r)\|_{X}=\sup _{[0,1[} \frac{|v(r)|}{\phi(r)}
$$


8 Radial solutions for a nonlocal boundary value problem

in the Banach space

$$
X=\left\{v \in C([0,1]): \frac{|v(r)|}{\phi(r)} \text { bounded }\right\}
$$

Then, as stated before, we can write problem (3.1)-(3.3) as $v=S v$, where

$$
S(v)(r)=\int_{0}^{1} G(r, t) f\left(v(t), \omega_{n} \int_{0}^{1} s^{n-1} g(v(s)) d s\right) d t, \quad \text { for } v \in X
$$

Let $T$ denote the operator introduced in Section 2, with $p(s)=s^{n-1}$. This operator acts in $C[0,1]$. Let $K$ be the restriction of $T$ to $X$ and $v \in X$. Since

$$
\begin{gathered}
|v(t)| \leq\|v\|_{X} \phi(t), \\
\int_{0}^{1} G(r, t) \phi(t) d t=\frac{\phi(r)}{\lambda_{1}}
\end{gathered}
$$

we have

$$
|K(v)(r)| \leq \int_{0}^{1} G(r, t)|v(t)| d t \leq\|v\|_{X} \int_{0}^{1} G(r, t) \phi(t) d t
$$

so that

$$
\frac{|K(v)(r)|}{\phi(r)} \leq \frac{\|v\|_{X}}{\lambda_{1}}
$$

Taking the least upper bound in the left-hand side of the last inequality, we obtain

$$
\|K(v)\|_{X} \leq \frac{\|v\|_{X}}{\lambda_{1}}
$$

This estimate, which is the main reason to work in the functional space $X$, will be used in the proof of Theorem 3.2 in a crucial way.

Lemma 3.3. The operator $S: X \rightarrow X$ is completely continuous.

Proof. Since the embedding $i_{1}: X \rightarrow C[0,1]$ is continuous, the Nemytskii operator $N$ : $X \rightarrow C[0,1]$ given, for each $v \in X$, by

$$
N(v)=f\left(v, \omega_{n} \int_{0}^{1} s^{n-1} g(v(s)) d s\right)
$$

is continuous. Moreover it takes bounded sets into bounded sets.

Now let us consider the following decomposition of $T$ :

$$
C[0,1] \stackrel{T_{*}}{\longrightarrow} C_{*}^{2}[0,1] \stackrel{i_{2}}{\longrightarrow} C_{*}^{1}[0,1] \stackrel{i_{3}}{\longrightarrow} X,
$$


where

$$
\begin{aligned}
& C_{*}^{2}[0,1]=\left\{u \in C^{2}[0,1]: u^{\prime}(0)=u(1)=0\right\}, \\
& C_{*}^{1}[0,1]=\left\{u \in C^{1}[0,1]: u(1)=0\right\},
\end{aligned}
$$

$i_{2}, i_{3}$ are embeddings, and $T_{*}$ is the operator $T$ acting between those two spaces.

The operator $\left(T_{*}\right)^{-1}$ takes $u$ into $-u^{\prime \prime}-((n-1) / r) u^{\prime}$; it is obviously linear continuous and bijective and, therefore, using the open map theorem, we get that $T_{*}$ is continuous. The embedding $i_{2}$ is a well-known completely continuous operator and using L'Hospital's rule we can prove that $i_{3}$ is also continuous. Since $S=i_{3} i_{2} T_{*} i_{1}$, the conclusion of the lemma is now straightforward.

Proof of Theorem 3.2. The proof is similar to that of Theorem 3.1 and so we only outline it. If $f\left(0, \omega_{n} g(0) / n\right)=0$, then $v \equiv 0$ is obviously a fixed point of the operator $S$, so let us suppose that $f\left(0, \omega_{n} g(0) / n\right)>0$. Then there exist positive constants $M$ and $\delta$ such that

$$
f\left(v(t), \omega_{n} \int_{0}^{1} s^{n-1} g(v(s)) d s\right) \geq M, \quad \forall\|v\|_{X} \leq \delta .
$$

A simple computation yields

$$
\|S v\|_{X} \geq M \sup _{r \in] 0,1[} \int_{0}^{1} \frac{G(r, t)}{\phi(r)} d t=M \epsilon,
$$

if $\|v\|_{X} \leq \delta$, where we have set $\epsilon:=\sup _{r \in] 0,1[} \int_{0}^{1}(G(r, t) / \phi(r)) d t$.

If we define $\Omega_{1}=\left\{v \in X \mid\|v\|_{X}<\min (M \epsilon / 2, \delta)\right\}$, in $\partial \Omega_{1}$ we have

$$
\|S v\|_{X} \geq M \epsilon>\|v\|_{X}
$$

Defining $\Omega_{2}=\left\{v \in X \mid\|v\|_{X}<\|T B\|_{X} /\left(1-A^{\prime} / \lambda_{1}\right)\right\}$ with $A<A^{\prime}<\lambda_{1}$, then for $v \in$ $P \cap \partial \Omega_{2}$ we have (using the positivity of $T$ and the estimate (3.32))

$$
\begin{aligned}
\|S v\|_{X} & \leq\|T(A v+B)\|_{X} \leq\|A K v\|_{X}+\|T B\|_{X} \\
& <\frac{A^{\prime} / \lambda_{1}\|T B\|_{X}}{1-A^{\prime} / \lambda_{1}}+\frac{\|T B\|_{X}-A^{\prime} / \lambda_{1}\|T B\|_{X}}{1-A^{\prime} / \lambda_{1}}=\|v\|_{X} .
\end{aligned}
$$

Applying Krasnoselskii's fixed point Theorem 2.3 (compression version) we find a fixed point of $S$, and therefore a positive radial solution of (1.1).

In both theorems above, as mentioned in [5], the condition on $f$ does not depend on the second variable, and, therefore, nothing is restraining the behaviour of $g$. The arguments used there are also valid for the same problem with $f(v(r), \alpha(v))$, for any continuous functional $\alpha$ in $X$.

A similar procedure allows us to prove a result in the spirit of the one considered in [5] where $g$ is restrained, but the condition on $f$ is weakened. 
Theorem 3.4. Let $f: \mathbb{R}^{+} \times \mathbb{R} \rightarrow \mathbb{R}^{+}$and $g: \mathbb{R}^{+} \rightarrow \mathbb{R}$ be continuous functions.

Suppose there exist positive constants $A<\lambda_{1}, B, C, D, p$, and $q$ with $p q \leq 1$ such that

$$
\begin{gathered}
f(v, y) \leq A v+B+C|y|^{p} \quad \forall v \geq 0, y \in \mathbb{R}, \\
|g(v)| \leq D|v|^{q} \quad \forall v \in \mathbb{R},
\end{gathered}
$$

where $\phi$ is the eigenfunction associated with $\lambda_{1}$.

Then the problem (1.1) has a positive radial solution.

Remark 3.5. We could have considered in (3.1) a right-hand side of the form $f\left(r, v(r), \omega_{n}\right.$ $\left.\int_{0}^{1} s^{n-1} g(v(s)) d s\right)$, continuous in $[0,1] \times \mathbb{R} \times \mathbb{R}$. Indeed we might even work with a nonlinear nonnegative function $f(r, v, w)$ continuous in $(v, w)$ for a.e. $r \in[0,1]$, and measurable in $r$ for all $(v, w) \in \mathbb{R} \times \mathbb{R}$. However in this case, defining

$$
L_{k}^{p}(0,1)=\{u: u \text { is measurable in }] 0,1\left[, \int_{0}^{1} s^{k}|u(s)| d s<+\infty\right\}
$$

we should confine ourselves to $L_{n-1}^{p}(0,1)$ Carathéodory functions $f$, that is,

$$
\forall M>0 \quad \sup _{|v|+|w| \leq M}|f(\cdot, v, w)| \in L_{n-1}^{p}(0,1)
$$

where $p>n$ is fixed.

Under this restriction, it can still be shown that the analogue of Lemma 3.3 holds, because we can obtain an analogue of $T$ acting compactly from $L_{n-1}^{p}(0,1)$ to $C_{*}^{1}[0,1]$.

\section{Lower and upper solutions and monotone approximation}

We will now apply the lower and upper solution method to find solutions of the boundary value problem (3.1)-(3.3).

We should point that in [10, page 695] a monotone method approach using lower and upper solutions is applied to an epidemic problem with diffusion. The problem considered in there is a second-order system of two PDE with a nonlocal term, under assumptions related to those we use below (in particular a Lipschitz condition) and where uniqueness is obtained as well.

We will use two different types of conditions concerning the given functions $f$ and $g$, and construct monotone convergent sequences to solutions of the problem.

Let us define the linear operator

$$
L u(r)=-u^{\prime \prime}(r)-\frac{n-1}{r} u^{\prime}(r)+\lambda u(r)
$$

Lemma 4.1. Let $\lambda \geq 0$, and $\left.u \in C^{1}[0,1] \cap C^{2}\right] 0,1[$ be such that $\operatorname{Lu}(r) \geq 0$ in $\left.] 0,1\right], u^{\prime}(0) \leq$ 0 and $u(1) \geq 0$. Then $u(r) \geq 0$ for all $r \in[0,1]$. 
Proof. Towards a contradiction, assume that $u\left(r_{0}\right)<0$ for some $\left.r_{0} \in\right] 0,1$ [. There are two cases to consider:

(i) $u(r)<0$ in some interval $] c, d[\subset[0,1]$, with $u(c)=u(d)=0$.

Let us consider first the case where $\lambda>0$. Then there must exist $p \in] c, d[$ such that $u^{\prime}(p)=0$ and $u^{\prime \prime}(p) \geq 0$, and since $u(p)<0$, we get $L u(p)<0$, which is a contradiction.

If $\lambda=0$, integrating in $[c, d]$, we get the contradiction

$$
0<d^{n-1} u^{\prime}(d)-c^{n-1} u^{\prime}(c) \leq 0
$$

(ii) $u(r)<0$ in some interval $[0, c[\subset[0,1]$, with $u(c)=0$.

If $u^{\prime}(0)<0$, the same argument applies. If $u^{\prime}(0)=0$, integrating in $[0, c]$, we get

$$
0>-c^{n-1} u^{\prime}(c)+\lambda \int_{0}^{c} r^{n-1} u(r) d r \geq 0 .
$$

From now on we assume that $f: \mathbb{R} \times \mathbb{R} \rightarrow \mathbb{R}$ and $g: \mathbb{R} \rightarrow \mathbb{R}$ are continuous functions. Consider the boundary value problem

$$
\begin{gathered}
-u^{\prime \prime}(r)-\frac{n-1}{r} u^{\prime}(r)=f\left(u(r), \omega_{n} \int_{0}^{1} s^{n-1} g(u(s)) d s\right) \quad \text { for } 0<r \leq 1, \\
u^{\prime}(0)=0=u(1) .
\end{gathered}
$$

We say that $\alpha(r)$ is a lower solution of (4.4)-(4.5) if

$$
\begin{gathered}
-\alpha^{\prime \prime}(r)-\frac{n-1}{r} \alpha^{\prime}(r) \leq f\left(\alpha(r), \omega_{n} \int_{0}^{1} s^{n-1} g(\alpha(s)) d s\right) \quad \text { for } 0<r \leq 1, \\
\alpha^{\prime}(0) \geq 0, \quad \alpha(1) \leq 0 .
\end{gathered}
$$

A function $\beta$ satisfying the reversed inequalities is called an upper solution.

Let $\alpha_{0}$ be a lower solution and $\beta_{0}$ an upper solution of (4.4)-(4.5). Consider the restriction $L_{0}$ of the operator $L$ to the subspace $\left\{u \in C^{1}[0,1] \cap C^{2}\right] 0,1\left[: u^{\prime}(0)=u(1)=0\right\}$. With the notations above, to get a solution of the problem (4.4)-(4.5) is equivalent to find a fixed point of the completely continuous operator in $C[0,1]$,

$$
\Phi u \equiv L_{0}^{-1}\left(f\left(u, \omega_{n} \int_{0}^{1} s^{n-1} g(u(s)) d s\right)+\lambda u\right) .
$$

Let us define

$$
R_{f}\left(u, v_{1}, v_{2}\right)=\frac{f\left(u, v_{2}\right)-f\left(u, v_{1}\right)}{v_{2}-v_{1}}, \quad R_{g}\left(u_{1}, u_{2}\right)=\frac{g\left(u_{2}\right)-g\left(u_{1}\right)}{u_{2}-u_{1}} .
$$

LEMmA 4.2. Let $\alpha_{0}$ be a lower solution and $\beta_{0}$ an upper solution of (4.4)-(4.5) such that $\alpha_{0} \leq \beta_{0}$ in $[0,1]$. Suppose $f(u, v)$ is such that

$$
f\left(u_{2}, v\right)-f\left(u_{1}, v\right) \geq-\lambda\left(u_{2}-u_{1}\right)
$$


12 Radial solutions for a nonlocal boundary value problem

for some $\lambda \geq 0, v \in \mathbb{R}, u_{1}, u_{2}$ such that for some $r \in[0,1], \alpha_{0}(r) \leq u_{1} \leq u_{2} \leq \beta_{0}(r)$, and $R_{f}$, $R_{g}$ have the same sign for all $u_{1}, u_{2}$ such that $\alpha_{0}(r) \leq u_{1}, u_{2} \leq \beta_{0}(r)$ for some $r \in[0,1]$.

Then, for any two functions $u_{1}(r), u_{2}(r) \in C[0,1]$ such that

$$
\alpha_{0}(r) \leq u_{1}(r) \leq u_{2}(r) \leq \beta_{0}(r)
$$

the following inequality holds:

$$
\Phi u_{1}(r) \leq \Phi u_{2}(r)
$$

Proof. The Green's function $G_{\lambda}$ associated with $L_{0}$ is nonnegative according to Lemma 4.1. We have

$$
\begin{aligned}
\Phi u_{2}(r) & -\Phi u_{1}(r) \\
= & \int_{0}^{1} G_{\lambda}(r, t)\left[f\left(u_{2}, \omega_{n} \int_{0}^{1} s^{n-1} g\left(u_{2}(s)\right) d s\right)-f\left(u_{1}, \omega_{n} \int_{0}^{1} s^{n-1} g\left(u_{2}(s)\right) d s\right)\right] d t \\
& +\int_{0}^{1} G_{\lambda}(r, t)\left[f\left(u_{1}, \omega_{n} \int_{0}^{1} s^{n-1} g\left(u_{2}(s)\right) d s\right)-f\left(u_{1}, \omega_{n} \int_{0}^{1} s^{n-1} g\left(u_{1}(s)\right) d s\right)\right] d t \\
& +\int_{0}^{1} G_{\lambda}(r, t) \lambda\left(u_{2}-u_{1}\right) d t \\
\geq & \int_{0}^{1} G_{\lambda}(r, t)\left[-\lambda\left(u_{2}-u_{1}\right)+\lambda\left(u_{2}-u_{1}\right)\right] d t \geq 0 .
\end{aligned}
$$

Remark 4.3. Clearly if $f$ and $g$ are $C^{1}$ functions, the hypotheses of the last theorem are satisfied provided that

$$
\frac{\partial f}{\partial u} \geq-\lambda, \quad \frac{\partial f}{\partial v}, \frac{\partial g}{\partial u} \text { have the same sign. }
$$

Theorem 4.4. Suppose that $f$ and $g$ satisfy the assumptions of Lemma 4.2. Let $\alpha_{0}, \beta_{0}$ be lower and upper solutions, respectively, of (4.4)-(4.5). Setting

$$
\alpha_{n+1}=\Phi \alpha_{n}, \quad \beta_{n+1}=\Phi \beta_{n}, \quad \forall n \in \mathbb{N}_{0},
$$

then

$$
\alpha_{0} \leq \alpha_{1} \leq \cdots \leq \alpha_{n} \leq \cdots \leq \beta_{n} \leq \cdots \leq \beta_{1} \leq \beta_{0} .
$$

The monotone bounded sequences $\left(\alpha_{n}\right)_{n \in \mathbb{N}_{0}}$ and $\left(\beta_{n}\right)_{n \in \mathbb{N}_{0}}$ defined above are convergent in $C[0,1]$, respectively, to the minimal and maximal solutions of (4.4)-(4.5) in the interval $\left[\alpha_{0}, \beta_{0}\right]$. 
Proof. Since

$$
\begin{aligned}
& L \alpha_{1}=f\left(\alpha_{0}, \omega_{n} \int_{0}^{1} s^{n-1} g\left(\alpha_{0}(s)\right) d s\right)+\lambda \alpha_{0}, \\
& L \alpha_{0} \leq f\left(\alpha_{0}, \omega_{n} \int_{0}^{1} s^{n-1} g\left(\alpha_{0}(s)\right) d s\right)+\lambda \alpha_{0},
\end{aligned}
$$

we have

$$
L\left(\alpha_{1}-\alpha_{0}\right) \geq 0, \quad\left(\alpha_{1}-\alpha_{0}\right)^{\prime}(0) \leq 0, \quad\left(\alpha_{1}-\alpha_{0}\right)(1) \geq 0,
$$

and therefore, by Lemma 4.1, we have $\alpha_{0} \leq \alpha_{1}$.

Using similar argumets, we can prove that $\alpha_{1} \leq \beta_{0}$.

We are now able to apply Lemma 4.2 to $\alpha_{0}$ and $\alpha_{1}$ which gives $\alpha_{1} \leq \alpha_{2}$. By iteration of this argument, we prove that $\left(\alpha_{n}\right)_{n \in \mathbb{N}_{0}}$ is an increasing sequence and stays below $\beta_{0}$. Analogously, we prove that $\left(\beta_{n}\right)_{n \in \mathbb{N}_{0}}$ is a decreasing sequence so that

$$
\alpha_{0} \leq \alpha_{1} \leq \cdots \leq \alpha_{n} \leq \cdots \leq \beta_{n} \leq \cdots \leq \beta_{1} \leq \beta_{0} .
$$

Concerning the convergence of the sequences, as the cone of positive functions in $C[0,1]$ is normal ( since $0 \leq u \leq v$ implies $\|u\| \leq\|v\|$ ), we can use the standard argument ([14, page 283]), which gives the convergence of this iteration method to fixed points of $\Phi$, and these are exactly the smallest and largest fixed points in $\left[\alpha_{0}, \beta_{0}\right] \subset C[0,1]$.

Example 4.5. Let us consider the nonlocal differential equation

$$
-u^{\prime \prime}(r)-\frac{2}{r} u^{\prime}(r)=\frac{4}{3} \pi e^{u} \int_{0}^{1} s^{2}(u(s)+1) d s
$$

with boundary conditions $u^{\prime}(0)=u(1)=0$.

In this case we have $n=3, f(u, v)=e^{u} v$ and $g(u)=(u+1) / 3$.

Consider $\alpha_{0} \equiv 0$ and $\beta_{0}=1-r$. Then

$$
-\alpha_{0}^{\prime \prime}(r)-\frac{2}{r} \alpha_{0}^{\prime}(r)=0 \leq \frac{4}{9} \pi=\frac{4}{3} \pi e^{0} \int_{0}^{1} s^{2} d s
$$

and $\alpha_{0}^{\prime}(0)=\alpha_{0}(1)=0$, so $\alpha_{0}$ is a lower solution of (4.5).

For $r \in[0,1]$ we have

$$
-\left(r^{2} \beta_{0}^{\prime}\right)^{\prime}=2 r \geq \frac{5}{9} \pi r^{2} e^{1-r}=\frac{4}{3} \pi r^{2} e^{1-r} \int_{0}^{1} s^{2}(1-s+1) d s
$$

$\beta_{0}^{\prime}(0)=-1$ and $\beta_{0}(1)=0$. Therefore $\beta_{0}$ is an upper solution of $(4.5)-(4.19)$.

The conditions in the Theorem 4.4 are satisfied for $\alpha_{0}$ and $\beta_{0}$, so there exists a solution $u$ of (4.5)-(4.19), such that

$$
0 \leq u(r) \leq 1-r, \quad \forall r \in[0,1] .
$$


14 Radial solutions for a nonlocal boundary value problem

This solution is the limit of a monotone sequence constructed as in the statement of the theorem.

Let us now try another approach using the lower and upper solution method, where we drop a part of the monotonicity assumptions.

Lemma 4.6. Suppose that $\left.u \in C^{1}[0,1] \cap C^{2}\right] 0,1[$ satisfies

$$
-u^{\prime \prime}(r)-\frac{n-1}{r} u^{\prime}(r)+\lambda u(r)+M \int_{0}^{1} s^{n-1}|u(s)| d s \geq 0
$$

for some $\lambda, M>0$ such that $\lambda+M<1$ and $u^{\prime}(0) \leq 0, u(1) \geq 0$. Then $u(r) \geq 0$ for all $r \in$ $[0,1]$.

Proof. Suppose by contradiction that there exists a function $u_{0}$ that satisfies the assumptions above and is negative at some point.

Normalising $\mathcal{u}_{0}$, we can assume that $\int_{0}^{1} s^{n-1}\left|\mathcal{u}_{0}(s)\right| d s=1$ without loss of generality, which implies that $\left\|r^{n-1} u_{0}(r)\right\|_{\infty} \geq 1$.

Let us consider the auxiliary problem

$$
-w^{\prime \prime}(r)-\frac{n-1}{r} w^{\prime}(r)+M=0, \quad w^{\prime}(0)=w(1)=0 .
$$

which is equivalent to

$$
\left(r^{n-1} w^{\prime}(r)\right)^{\prime}=r^{n-1} M, \quad w^{\prime}(0)=w(1)=0 .
$$

Integrating (4.25), we get

$$
w(r)=\frac{M}{2 n}\left(r^{2}-1\right) \leq 0 .
$$

As $u_{0}$ satisfies

$$
-u_{0}^{\prime \prime}(r)-\frac{n-1}{r} u_{0}^{\prime}(r)+\lambda u_{0}(r)+M \geq 0,
$$

with $u_{0}^{\prime}(0) \leq 0, u_{0}(1) \geq 0$, we have

$$
\begin{gathered}
-\left(u_{0}-w\right)^{\prime \prime}-\frac{n-1}{r}\left(u_{0}-w\right)^{\prime}+\lambda\left(u_{0}-w\right) \geq-\lambda w, \\
\left(u_{0}-w\right)^{\prime}(0) \leq 0,\left(u_{0}-w\right)(1) \geq 0,
\end{gathered}
$$

and, therefore, applying Lemma 4.1 , we get $u_{0} \geq w$.

We can easily see that $r^{n-1} u_{0}(r) \geq r^{n-1} w(r) \geq-M / 2 n>-1$, so the fact that $\left\|r^{n-1} u_{0}(r)\right\|_{\infty} \geq 1$ insures that there exists $a>0$ such that $u_{0}(a) \geq 1 / a^{n-1}$.

If $u_{0}$ is negative at $b>a$, there exists $c \in$ ] $a, b$ [ such that $u_{0}(c)=0$ (we can assume that $\left.u_{0}^{\prime}(b)=0\right)$. Using Lagrange's theorem, there exists $d \in[a, c]$ such that $u_{0}^{\prime}(d) \leq-1$ / $a^{n-1}$. As $d \geq a$, we have $d^{n-1} u_{0}^{\prime}(d) \leq-1$ and therefore there exists $e \in[d, b]$ such that $\left.\left(r^{n-1} u_{0}^{\prime}(r)\right)^{\prime}\right|_{r=e} \geq 1$, (we can take $e$ such that $\left.e^{n-1} u_{0}(e)<1\right)$. 
If $u$ is negative at $b<a$, there exists $c<a$ such that $u_{0}(c)=0$. As $u_{0}(a)>1$, there exists $d \in] c, a\left[\right.$ such that $u_{0}^{\prime}(d) \geq 1$. Considering the boundary condition $u_{0}^{\prime}(0) \leq 0$, there exists $e \in\left[0, d\left[\right.\right.$ such that $u_{0}^{\prime}(e)=0$ and $u_{0}^{\prime}(r)>0$ for all $\left.\left.r \in\right] e, d\right]$. Therefore there exists $f \in$ $[e, d]$ such that $u_{0}^{\prime \prime}(f) \geq 1$ and $u_{0}^{\prime}(f)>0$ (we can take $f$ such that $f^{n-1} u_{0}(f)<1$ ).

In both cases, we know that for some $r_{0}$ we have $\left.\left(r^{n-1} u_{0}^{\prime}(r)\right)^{\prime}\right|_{r=r_{0}} \geq 1$, and $r_{0}^{n-1} u_{0}\left(r_{0}\right)<1$. Therefore we would get

$$
-\left.\left(r^{n-1} u_{0}^{\prime}(r)\right)^{\prime}\right|_{r=r_{0}}+\lambda r_{0}^{n-1} u_{0}\left(r_{0}\right)+M \leq-1+\lambda+M<0
$$

which is a contradiction.

For a given function $u(r) \in C[0,1]$, consider the boundary value problem

$$
-v^{\prime \prime}(r)-\frac{n-1}{r} v^{\prime}(r)+\lambda v(r)=f\left(u(r), \omega_{n} \int_{0}^{1} s^{n-1} g(v(s)) d s\right)+\lambda u(r),
$$

with $v^{\prime}(0)=0=v(1)$. Using the operator $L$ defined in the beginning of this section, this equation is equivalent to the fixed point equation in $C[0,1]$,

$$
v=L_{0}^{-1}\left(f\left(u, \omega_{n} \int_{0}^{1} s^{n-1} g(v(s)) d s\right)+\lambda u\right) \equiv \Phi_{u} v .
$$

Remark 4.7. Using a comparison method as the one in the proof of Lemma 4.6, we get $\left\|L_{0}^{-1}\right\| \leq 1 / 2 n$ in $C[0,1]$.

Lemma 4.8. If $f(u, v)$ is $k_{1}$-Lipschitz in $v, g$ is $k_{2}$-Lipschitz, and $k_{1} k_{2} \omega_{n}<2 n^{2}$, then $\Phi_{u}$ has a unique fixed point.

Proof. We have

$$
\begin{aligned}
\left|\Phi_{u} v_{2}(r)-\Phi_{u} v_{1}(r)\right| & \leq \frac{1}{2 n} k_{1}\left|\omega_{n} \int_{0}^{1} s^{n-1} g\left(v_{2}(s)\right) d s-\omega_{n} \int_{0}^{1} s^{n-1} g\left(v_{1}(s)\right) d s\right| \\
& \leq \frac{1}{2 n} k_{1} k_{2} \omega_{n} \int_{0}^{1} s^{n-1}\left|v_{2}(s)-v_{1}(s)\right| d s \leq \frac{k_{1} k_{2} \omega_{n}}{2 n^{2}}\left\|v_{2}-v_{1}\right\|_{\infty}
\end{aligned}
$$

so that

$$
\left\|\Phi_{u} v_{2}(r)-\Phi_{u} v_{1}(r)\right\|_{\infty} \leq \frac{k_{1} k_{2} \omega_{n}}{2 n^{2}}\left\|v_{2}-v_{1}\right\|_{\infty}
$$

and therefore $\Phi_{u}$ is a contraction mapping.

Lemma 4.9. Let $f$ and $g$ be functions defined as in Lemma 4.8, $\lambda>0$ such that $k_{1} k_{2} \omega_{n}+\lambda<$ 1 , and suppose that

$$
f\left(u_{2}, v\right)-f\left(u_{1}, v\right) \geq-\lambda\left(u_{2}-u_{1}\right),
$$

for all $r \in[0,1], v \in \mathbb{R}$, and $u_{1} \leq u_{2}$.

Let $u_{1}(r) \leq u_{2}(r)$ be two given functions defined in $[0,1]$ and $v_{1}(r), v_{2}(r)$ the two respective solutions of (4.31). Then $v_{1}(r) \leq v_{2}(r)$. 
16 Radial solutions for a nonlocal boundary value problem

Proof. We have

$$
\begin{aligned}
-\left(v_{2}-v_{1}\right)^{\prime \prime}-\frac{n-1}{r}\left(v_{2}-v_{1}\right)^{\prime}+\lambda\left(v_{2}-v_{1}\right) \\
=\lambda\left(u_{2}-u_{1}\right)+f\left(u_{2}, \omega_{n} \int_{0}^{1} s^{n-1} g\left(v_{2}\right) d s\right)-f\left(u_{1}, \omega_{n} \int_{0}^{1} s^{n-1} g\left(v_{2}\right) d s\right) \\
\quad+f\left(u_{1}, \omega_{n} \int_{0}^{1} s^{n-1} g\left(v_{2}\right) d s\right)-f\left(u_{1}, \omega_{n} \int_{0}^{1} s^{n-1} g\left(v_{1}\right) d s\right) \\
\geq-k_{1} k_{2} \omega_{n} \int_{0}^{1} s^{n-1}\left|v_{2}-v_{1}\right| d s .
\end{aligned}
$$

The conclusion follows from Lemma 4.6.

Theorem 4.10. Suppose that $f(u, v)$ is $k_{1}$-Lipschitz in $v, g$ is $k_{2}$-Lipschitz. Suppose that for some $\lambda>0$ such that $k_{1} k_{2} \omega_{n}+\lambda<1$,

$$
f\left(u_{2}, v\right)-f\left(u_{1}, v\right) \geq-\lambda\left(u_{2}-u_{1}\right)
$$

for all $v \in \mathbb{R}$, and $u_{1} \leq u_{2}$. Let $\alpha_{0}$ and $\beta_{0}$ be a lower solution and an upper solution of (4.4)(4.5), respectively, with $\alpha_{0} \leq \beta_{0}$ in $[0,1]$. Taking $\left(\alpha_{n}\right)_{n \in \mathbb{N}_{0}}$ and $\left(\beta_{n}\right)_{n \in \mathbb{N}_{0}}$ such that, according to Lemma 4.8,

$$
\alpha_{n+1}=\Phi_{\alpha_{n}} \alpha_{n+1}, \quad \beta_{n+1}=\Phi_{\beta_{n}} \beta_{n+1}, \quad \forall n \in \mathbb{N}_{0}
$$

then

$$
\alpha_{0} \leq \alpha_{1} \leq \cdots \leq \alpha_{n} \leq \cdots \leq \beta_{n} \leq \cdots \leq \beta_{1} \leq \beta_{0} .
$$

The monotone bounded sequences $\left(\alpha_{n}\right)_{n \in \mathbb{N}_{0}},\left(\beta_{n}\right)_{n \in \mathbb{N}_{0}}$ defined above are convergent in $C[0,1]$ to solutions of (4.4)-(4.5).

Proof. The computation used here is similar to another one used in [9]. We have

$$
\begin{aligned}
L\left(\alpha_{1}-\alpha_{0}\right) & \geq f\left(\alpha_{0}, \omega_{n} \int_{0}^{1} s^{n-1} g\left(\alpha_{1}(s)\right) d s\right)-f\left(\alpha_{0}, \omega_{n} \int_{0}^{1} s^{n-1} g\left(\alpha_{0}(s)\right) d s\right) \\
& \geq-k_{1} k_{2} \omega_{n} \int_{0}^{1}\left|\alpha_{1}(s)-\alpha_{0}(s)\right| d s
\end{aligned}
$$

with

$$
\left(\alpha_{1}-\alpha_{0}\right)^{\prime}(0) \leq 0, \quad\left(\alpha_{1}-\alpha_{0}\right)(1) \geq 0
$$

and, therefore, using Lemma 4.6, we get $\alpha_{0} \leq \alpha_{1}$. Let us prove that $\alpha_{1} \leq \beta_{0}$. This comes 
from

$$
\begin{aligned}
L\left(\beta_{0}-\alpha_{1}\right) & \geq f\left(\beta_{0}, \omega_{n} \int_{0}^{1} s^{n-1} g\left(\beta_{0}(s)\right) d s\right)+\lambda \beta_{0}-f\left(\alpha_{0}, \omega_{n} \int_{0}^{1} s^{n-1} g\left(\beta_{0}(s)\right) d s\right) \\
& +f\left(\alpha_{0}, \omega_{n} \int_{0}^{1} s^{n-1} g\left(\beta_{0}(s)\right) d s\right)-f\left(\alpha_{0}, \omega_{n} \int_{0}^{1} s^{n-1} g\left(\alpha_{1}(s)\right) d s\right)-\lambda \alpha_{0} \\
& \geq-\lambda\left(\beta_{0}-\alpha_{0}\right)+\lambda\left(\beta_{0}-\alpha_{0}\right)-k_{1} k_{2} \omega_{n} \int_{0}^{1}\left|\beta_{0}(s)-\alpha_{1}(s)\right| d s \\
& =-k_{1} k_{2} \omega_{n} \int_{0}^{1}\left|\beta_{0}(s)-\alpha_{1}(s)\right| d s .
\end{aligned}
$$

Applying this lemma in the following iterations, we prove that

$$
\alpha_{0} \leq \alpha_{1} \leq \cdots \leq \alpha_{n} \leq \cdots \leq \beta_{n} \leq \cdots \leq \beta_{1} \leq \beta_{0}
$$

as in the proof of Theorem 4.4.

Concerning the convergence of the sequences, there is a slight difference from the usual method, because in each iteration we use a different operator. But, as

$$
\alpha_{n+1}(r)=L_{0}^{-1}\left(f\left(\alpha_{n}, \omega_{n} \int_{0}^{1} s^{n-1} g\left(\alpha_{n+1}(s)\right) d s\right)+\lambda \alpha_{n}\right)
$$

and $\left\|\alpha_{n}\right\|_{\infty} \leq \max \left(\left\|\alpha_{0}\right\|_{\infty},\left\|\beta_{0}\right\|_{\infty}\right)$, we have that $\left\|\alpha_{n+1}\right\|_{C^{1}}$ is bounded, and, therefore, using Àrzela-Ascoli Theorem, there exists a convergent subsequence of $\alpha_{n}$. Considering the monotonicity of $\alpha_{n}$, we get the conclusion by the standard argument.

Remark 4.11. It is not difficult to prove that the monotone sequences defined in Theorem 4.10 converge in fact to extremal solutions of the boundary value problem (4.4)-(4.5).

Example 4.12. Suppose that

$$
\liminf _{(a, b) \rightarrow\left(0^{+}, 0^{+}\right)} \frac{f\left(a,\left(\omega_{n} / n\right) g(b)\right)}{a}>\lambda_{1} .
$$

and there exists $k>0$ such that $f\left(k, \omega_{n} g(k) / n\right)<0$. Suppose in addition that $f$ and $g$ satisfy the assumptions of Theorem 4.10.

Then there exists a positive solution of (4.4)-(4.5). This solution may be approximated by monotone sequences. In fact, a simple calculation shows that for $\epsilon>0$ small enough, $\epsilon \phi$ is a positive lower solution of (4.4)-(4.5). The constant $k$ is clearly an upper solution. The statement follows.

\section{Acknowledgments}

This work was supported by Fundação para a Ciência e Tecnologia, projects POCTI/Mat/ 57258/2004, and POCTI-ISFL-1-209 (Centro de Matemática e Aplicações Fundamentais). 


\section{References}

[1] J. W. Bebernes and A. A. Lacey, Global existence and finite-time blow-up for a class of nonlocal parabolic problems, Advances in Differential Equations 2 (1997), no. 6, 927-953.

[2] N.-H. Chang and M. Chipot, On some mixed boundary value problems with nonlocal diffusion, Advances in Mathematical Sciences and Applications 14 (2004), no. 1, 1-24.

[3] C. De Coster and P. Habets, The lower and upper solutions method for boundary value problems, Handbook of Differential Equations, Elsevier, New York; North-Holland, Amsterdam, 2004, pp. 69-160.

[4] K. Deimling, Nonlinear Functional Analysis, Springer, Berlin, 1985.

[5] P. Fijałkowski and B. Przeradzki, On a radial positive solution to a nonlocal elliptic equation, Topological Methods in Nonlinear Analysis 21 (2003), no. 2, 293-300.

[6] P. Freitas and G. Sweers, Positivity results for a nonlocal elliptic equation, Proceedings of the Royal Society of Edinburgh. Section A. Mathematics 128 (1998), no. 4, 697-715.

[7] M. Gaudenzi, P. Habets, and F. Zanolin, Positive solutions of singular boundary value problems with indefinite weight, Bulletin of the Belgian Mathematical Society. Simon Stevin 9 (2002), no. 4, 607-619.

[8] J. M. Gomes and L. Sanchez, On a variational approach to some non-local boundary value problems, Applicable Analysis 84 (2005), no. 9, 909-925.

[9] D. Jiang, W. Gao, and A. Wan, A monotone method for constructing extremal solutions to fourthorder periodic boundary value problems, Applied Mathematics and Computation 132 (2002), no. 2-3, 411-421.

[10] C. V. Pao, Nonlinear Parabolic and Elliptic Equations, Plenum Press, New York, 1992.

[11] V. Smirnov, Cours de Mathématiques Supérieures, vol. 2, Mir, Moscoú, 1970.

[12] W. Walter, Ordinary Differential Equations, Graduate Texts in Mathematics, vol. 182, Springer, New York, 1998.

[13] Z. Yang, Positive solutions to a system of second-order nonlocal boundary value problems, Nonlinear Analysis. Theory, Methods \& Applications 62 (2005), no. 7, 1251-1265.

[14] E. Zeidler, Nonlinear Functional Analysis and Its Applications-I : Fixed-Point Theorems, Springer, New York, 1986.

Ricardo Enguiça: Área Científica de Matemática, Instituto Superior de Engenharia de Lisboa, Rua Conselheiro Emídio Navarro, 1-1950-062 Lisboa, Portugal

E-mail address: rroque@dec.isel.ipl.pt

Luís Sanchez: Faculdade de Ciências da Universidade de Lisboa, Avenida Professor Gama Pinto 2, 1649-003 Lisboa, Portugal

E-mail address: sanchez@ptmat.fc.ul.pt 